\title{
Proposed Inspection Method for Opposite-side Defect in Steel Plate Using Synthetic Magnetic Field with High and Low Excitation Frequencies
}

\author{
Saijiro Yoshioka, ${ }^{1}$ Akira Fujii, ${ }^{2}$ Makoto Tohara, ${ }^{3}$ and Yuji Gotoh ${ }^{4 *}$ \\ ${ }^{1}$ Department of Informatics and Mechanical Engineering, National Institute of Technology, \\ Toba College, 1-1 Ikegami, Toba, Mie 517-8501, Japan \\ ${ }^{2}$ Information Systems Department, Japan Organization for Employment of the Elderly, \\ Persons with Disabilities and Job Seekers, 3-1-2 Wakaba, Mihama-ku, Chiba 261-8558, Japan \\ ${ }^{3}$ Department of Technical Development, TOA Non-Destructive Inspection Co., Ltd, \\ 8-46 Shinsone, Kitakyushu, Kyusyu 800-0211, Japan \\ ${ }^{4}$ Department of Innovative Engineering, Faculty of Science and Technology, Oita University, \\ 700 Dannoharu, Oita 870-1192, Japan
}

(Received April 21, 2021; accepted June 24, 2021)

Keywords: 3D nonlinear FEM, nondestructive inspection, opposite-side defect in steel plate, ac synthetic magnetic field, minor loop magnetism property

A defect on the opposite-side of a steel plate is detected by an electromagnetic inspection method using a large static magnetic field and a small alternating magnetic field. However, in this inspection method, it is difficult for the inspection sensor to move because strong magnetic attraction is generated between the steel plate and the inspection sensor. To improve the practicality of this inspection method, it is necessary to weaken the magnetic attraction and improve the detection sensitivity. In this paper, an inspection method for opposite-side defects in a steel plate that uses an alternating magnetic field with high and low excitation frequencies is proposed. It is shown that the detection sensitivity of an opposite-side defect in a steel plate using the alternating magnetic field with the two excitation frequencies is higher than that using a large static magnetic field and a minute alternating magnetic field. The phenomenon underlying this technique is elucidated by analysis using 3D nonlinear finite-element method taking account of the hysteresis (minor loop) and eddy current.

\section{Introduction}

Electromagnetic inspection, ultrasonic inspection, and so forth are applied for the maintenance inspection of petrochemical plants. Generally, an electromagnetic inspection method capable of high-speed inspection is required for defect inspection on the opposite side of a large-diameter steel pipe with a thickness of about $2 \mathrm{~mm}$ in such plants. For this inspection, an electromagnetic inspection method using a large static magnetic field and a minute AC magnetic field is being studied. ${ }^{(1-4)}$ However, in this inspection method, a degaussing process is needed since large residual magnetism is generated inside the steel plate during the inspection. ${ }^{(5)}$

*Corresponding author: e-mail: goto-yuuji@oita-u.ac.jp https://doi.org/10.18494/SAM.2021.3380 
Moreover, it is also difficult for the inspection sensor to move because strong magnetic attraction is generated between the inspection steel plate and the inspection sensor. In this paper, an inspection method for opposite-side defects is proposed, in which a large static magnetic field is replaced with a large alternating magnetic field of low excitation frequency. Moreover, in this research, a steel plate with a thickness of $2 \mathrm{~mm}$ is examined assuming a large-diameter steel pipe. This method is investigated by a 3D nonlinear finite element method (FEM), taking account of the minor loop magnetism property in the steel plate, and a verification experiment is also performed.

\section{Inspection Model and Conditions}

Figure 1 shows the proposed model for inspecting a steel plate (SS400) for opposite-side defects. This inspection sensor is composed of a magnetic yoke with two excitation coils of high and low frequencies, and a search coil for the $x$-direction. Excitation coil-1 with a low frequency is set to $5 \mathrm{~Hz}$ with 142 ampere-turns, and excitation coil-2 with a high frequency is set to $500 \mathrm{~Hz}$ with 14.2 ampere-turns. The detection coil is wound on a thin stainless steel plate with a thickness of $0.75 \mathrm{~mm}$. The $x$-direction leakage flux $\left(B_{x}\right)$ of the surface on the steel plate is detected. $^{(6-10)}$ A basic experiment is conducted to confirm the inspection sensitivity of the proposed method and clarify the detection principle. Therefore, a steel plate having a thickness of $2 \mathrm{~mm}$ is used. An opposite-side defect is assumed to have the shape of a slit. The depth $\left(D_{d}\right)$ in the $z$-direction, width $\left(D_{w}\right)$ in the $x$-direction, and length $\left(D_{l}\right)$ in the $y$-direction of a defect are 1 , 0.5 , and $100 \mathrm{~mm}$, respectively. In this experiment and analysis, an opposite-side defect in the steel plate is detected at a pitch of $1 \mathrm{~mm}$ in the $x$-direction while keeping the $z$-direction distance (lift-off: $L_{o}$ ) between the sensor and the steel plate at $0.5 \mathrm{~mm}$. In the 3D nonlinear electromagnetic FEM analysis, the flux density in the detection coil at the peak value of the alternating magnetic

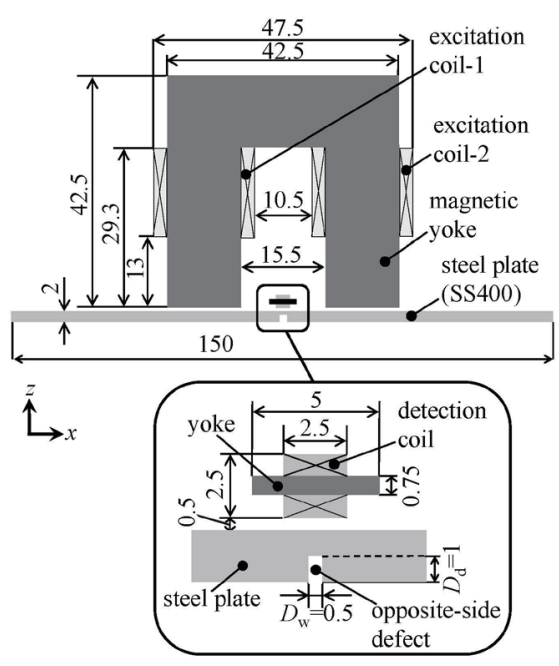

(a)

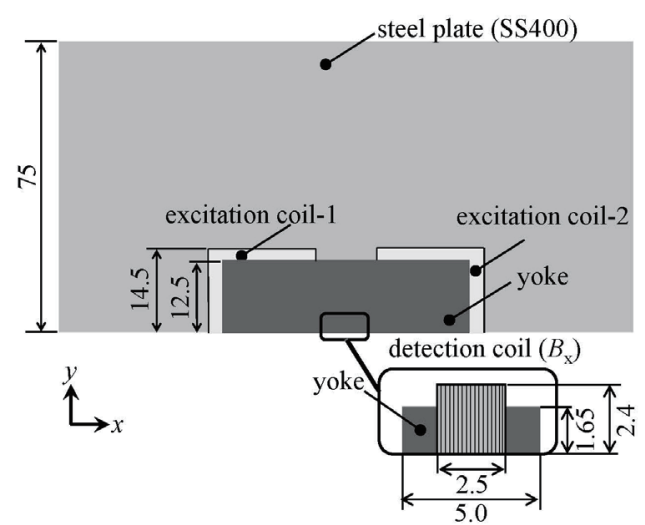

(b)

Fig. 1. Inspection model for opposite-side defect in SS400 steel plate. (a) $x-z$ plane and (b) $x-y$ plane. 
field of high frequency $(500 \mathrm{~Hz})$ is evaluated when the large alternating magnetic field of low frequency $(5 \mathrm{~Hz})$ is at the peak value. The conditions of 3D nonlinear FEM and the verification experiment are shown in Table 1 . The $z$-directions of the domain of the steel plate and the detection coil in the FEM analysis model are divided at a pitch of $0.2 \mathrm{~mm}$. In the analysis results shown later, the mesh division line is displayed.

\section{3D Nonlinear FEM Taking Account of Minor Loop}

\subsection{Method of analysis}

The flux density and eddy current density due to the large magnetic field with the lowfrequency excitation current and the minute magnetic field with the high-frequency excitation current are calculated by 3D FEM analysis considering the minor loop magnetization curve in the steel plate. ${ }^{(11)}$ Figure 2 shows the initial magnetization curve and the hysteresis loops of the examined SS400 steel plate obtained by measurement. First, only the large alternating magnetic field with the low frequency of $5 \mathrm{~Hz}$ is calculated up to the peak value of the sinusoidal wave in the steady state by 3D nonlinear FEM analysis using the initial magnetization curve in Fig. 2. Then, the minute alternating magnetic field with the high frequency of $500 \mathrm{~Hz}$ is added to the peak value of the large alternating magnetic field with the low frequency as shown in Fig. 3. The synthetic flux density in the steel plate is analyzed by 3D nonlinear FEM taking account of the minor loop magnetization characteristic using the hysteresis loops in Fig. 2. The basic equation

Table 1

Conditions of calculation and verification experiment using synthetic magnetic fields of low and high frequencies.

\begin{tabular}{|c|c|}
\hline Excitation coil-1 & 71 turns, $5 \mathrm{~Hz}, 2 \mathrm{~A}(\mathrm{rms})$ \\
\hline Excitation coil-2 & 71 turns, $500 \mathrm{~Hz}, 0.2 \mathrm{~A}(\mathrm{rms})$ \\
\hline Detection coil & $\begin{array}{l}60 \text { turns, width in } x \text {-direction: } 2.5 \mathrm{~mm} \text {, } \\
\text { thickness in } z \text {-direction: } 2.5 \mathrm{~mm} \text {, } \\
\text { length in } y \text {-direction: } 3.3 \mathrm{~mm}\end{array}$ \\
\hline Lift-off $\left(L_{o}\right)$ & $0.5 \mathrm{~mm}$ \\
\hline Specimen & $\begin{array}{l}\text { SS400 steel plate, } \\
\text { maximum relative permeability: } 1500 \\
\text { conductivity: } 7.505 \times 10^{6} \mathrm{~S} / \mathrm{m} \text {, } \\
\text { width in } x \text {-direction: } 150 \mathrm{~mm} \text {, } \\
\text { thickness in } z \text {-direction: } 2 \mathrm{~mm} \text {, } \\
\text { length in } y \text {-direction: } 150 \mathrm{~mm} \text {, } \\
\text { nonlinear calculation }\end{array}$ \\
\hline Opposite side defect & $\begin{array}{l}\text { width in } x \text {-direction }\left(D_{w}\right): 0.5 \mathrm{~mm} \text {, } \\
\text { depth in } z \text {-direction }\left(D_{d}\right): 1 \mathrm{~mm} \text {, } \\
\text { length in } y \text {-direction }\left(D_{l}\right): 100 \mathrm{~mm}\end{array}$ \\
\hline Magnetic yoke & $\begin{array}{l}\text { laminated stainless steel plate, } \\
\text { maximum relative permeability: } 10000 \\
\text { conductivity: } 0 \mathrm{~S} / \mathrm{m} \text {, } \\
\text { linear calculation }\end{array}$ \\
\hline Nodes and elements & 29008,25920 \\
\hline Convergence criterion & $\begin{array}{l}N-R \text { method: } 1.0 \times 10^{-6} \mathrm{~T} \text {, } \\
\text { Incomplete Cholesky Conjugate Gradient }(\mathrm{ICCG}) \text { method: } 1.0 \times 10^{-5}\end{array}$ \\
\hline
\end{tabular}




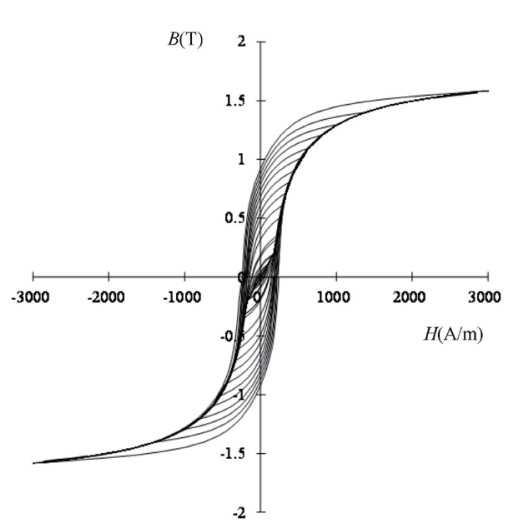

Fig. 2. Initial magnetization curve and the hysteresis curves of SS400 steel plate.

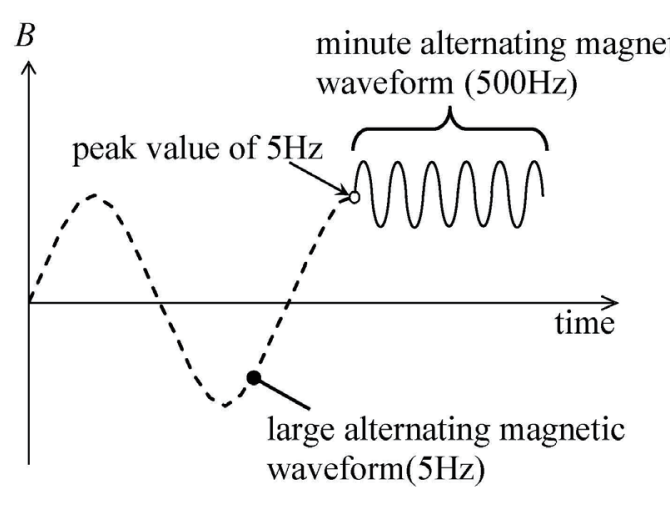

Fig. 3. Timing of addition of the minute alternating magnetic field with high frequency $(500 \mathrm{~Hz})$ to the peak value of the large alternating magnetic field with low frequency $(5 \mathrm{~Hz})$ in 3D FEM analysis.

of this analysis using the $\boldsymbol{A}-\phi$ method considering the minor loop magnetization curve is given by

$$
\begin{gathered}
\operatorname{rot}(v \operatorname{rot} \boldsymbol{A})=\boldsymbol{J}_{0}-\sigma\left(\frac{\partial \boldsymbol{A}}{\partial t}+\operatorname{grad} \phi\right) \\
\operatorname{div}\left\{-\sigma\left(\frac{\partial \boldsymbol{A}}{\partial t}+\operatorname{grad} \phi\right)\right\}=0,
\end{gathered}
$$

where $\boldsymbol{A}$ is the magnetic vector potential, $\phi$ is the scalar potential, $v$ is the reluctivity, $\boldsymbol{J}_{0}$ is the current density, and $\sigma$ is the conductivity. Figure 4 illustrates the calculation procedure for the minor loop magnetization curve in the steel plate. In the analysis considering the minor loop magnetization curve, the upper curve of the minor loop is first modeled by linear interpolation using the hysteresis loops shown in Fig. 2. ${ }^{(12)}$ Then, it is assumed that the obtained $H$ and $B$ are at point $b\left(H_{\min }, B_{\min }\right)$ on the upper minor curve as shown in Fig. 4. If the calculated flux density $B_{c}$ in the Newton-Raphson $(N-R)$ iteration is larger than $B_{\text {min }}$, then $B_{c}$ will be located at point $d\left(H_{d}, B_{c}\right)$ on the lower curve of the minor loop. $H_{d}$ on the lower minor curve is given by the following equation if the upper minor curve is symmetrical with respect to the center point $e$ :

$$
H_{d}=H_{f}+2\left(H_{e}-H_{f}\right) .
$$

$v_{d}$ and $\partial v_{d} / \partial B_{c}^{2}$, which are necessary in the $N-R$ iteration, are given by

$$
v_{d}=\frac{H_{d}}{B_{c}},
$$




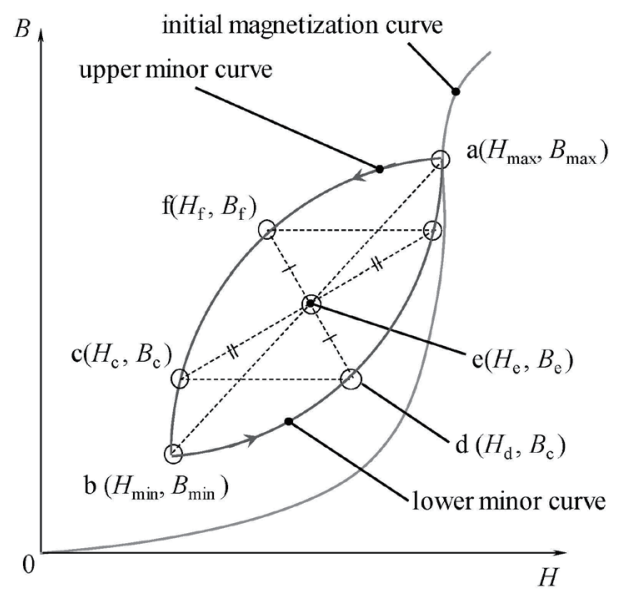

Fig. 4. Explanation of minor loop.

$$
\frac{\partial v_{d}}{\partial B_{c}^{2}}=\frac{1}{2 B_{c}^{2}}\left(\frac{\partial H_{g}}{\partial B_{g}}-v_{d}\right),
$$

where $\partial v_{d} / \partial B_{c}^{2}$ at point $d$ is obtained using the property that $\partial v_{d} / \partial B_{c}^{2}$ at point $d$ is equal to that at point $f$.

The minute alternating magnetic field is calculated up to the steady state by $3 \mathrm{D}$ nonlinear FEM analysis, taking account of the minor loop magnetization curve, using Eqs. (1)-(5).

\subsection{Results of analysis}

Figure 5 shows the vector distribution of the synthetic flux density of the large low frequency and minute high-frequency fields inside the steel plate with and without an opposite-side defect. The displayed magnetic flux distribution is the distribution at the peak of the 5 and $500 \mathrm{~Hz}$ waveforms. This figure shows that the flux density is concentrated in the layer between the opposite-side defect and the surface steel plate when there is a defect. If there is an opposite-side defect, the flux density in the surface layer of the steel plate is increased from 1.16 to 1.63T. Since the flux density in the surface layer of the steel plate approaches magnetic saturation when there is a defect, the leakage flux to the air domain on the surface of the steel plate is increased. The peak value of the flux density $B_{x}$ at the $500 \mathrm{~Hz}$ component inside the detection coil is increased from $1.319 \times 10^{-2}$ to $1.54 \times 10^{-2} \mathrm{~T}$ when there is a defect. The difference $\Delta B_{x}$ of the flux density in the detection coil with and without a defect is $0.221 \times 10^{-2} \mathrm{~T}$. Since the leakage flux is increased near the defect position, the opposite-side defect is detected from the flux density $B_{x}$ in the detection coil. 


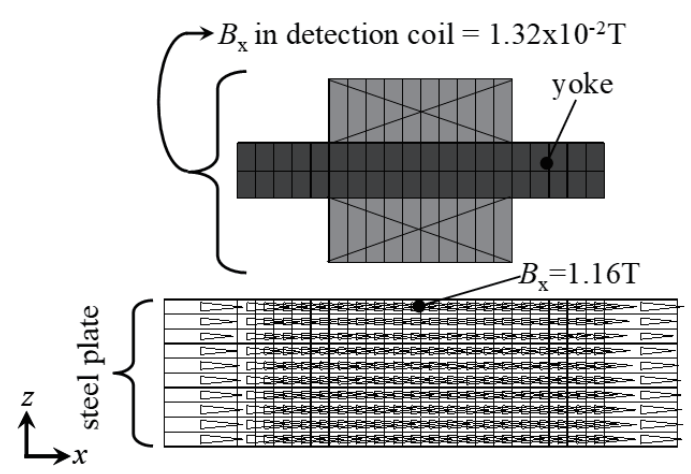

(a)

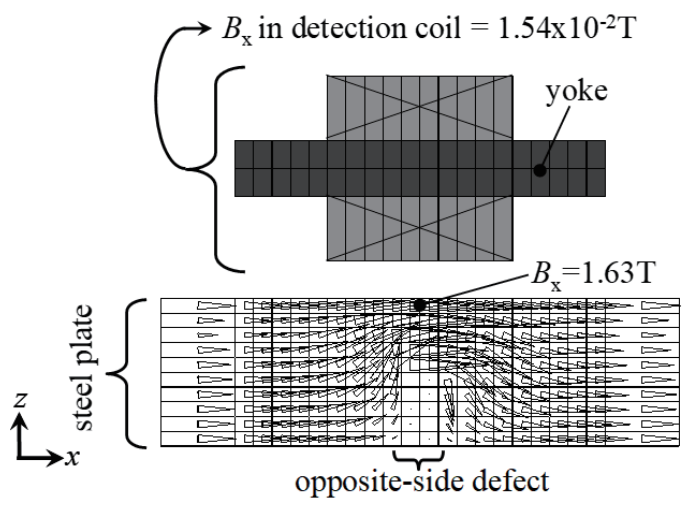

(b)

Fig. 5. Distribution of synthetic magnetic field of low $(5 \mathrm{~Hz})$ and high $(500 \mathrm{~Hz})$ excitation frequencies in the steel plate. (a) Without a defect and (b) with a defect.

\subsection{Comparison with synthetic magnetic field consisting of static and alternating magnetic fields}

In an actual plant, the electromagnetic inspection method using large static and minute alternating magnetic fields is used for opposite-side defect detection in a steel plate. ${ }^{(2,3)}$ Therefore, the detection sensitivity of an opposite-side defect is compared for a synthetic magnetic field consisting of a large static magnetic field and a minute alternating magnetic field and for a synthetic magnetic field consisting of a large magnetic field of low frequency and a minute magnetic field of high frequency. The inspection model using the large static magnetic field and the minute alternating magnetic field is the same as that in Fig. 1. The direct excitation current for the static magnetic field of excitation coil-1 is set to 142 ampere-turns, and the alternating excitation current of excitation coil-2 is set to 14.2 ampere-turns with a frequency of $500 \mathrm{~Hz}$. The inspection conditions of the calculation and experiment using the synthetic magnetic field consisting of the large static magnetic field and the minute alternating magnetic field with the high excitation frequency are shown in Table 2.

The flux density inside the steel plate when the large static magnetic field is applied to the steel plate is calculated by 3D nonlinear FEM analysis using the initial magnetization curve in Fig. 2. Then, the flux density $B_{x}$ of the synthetic magnetic field in the steel plate is analyzed up to the steady state by 3D nonlinear FEM analysis taking account of the minor loop magnetization curve, using Eqs. (1)-(5).

Figure 6 shows the vector distribution of the synthetic flux density of the large static and minute alternating magnetic fields inside the steel plate with and without an opposite-side defect. The displayed magnetic flux distribution is that when the AC waveform is at its peak. This figure shows that when there is an opposite-side defect in the steel plate, the flow of the flux density bypasses the defect and the flux density is concentrated in the layer between the defect and the surface steel plate. If there is an opposite-side defect, the flux density in the surface layer of the steel plate is increased from 1.24 to $1.59 \mathrm{~T}$. Therefore, the leakage flux to the air domain 
Table 2

Conditions of calculation and verification experiment for synthetic magnetic field consisting of large static magnetic field and minute alternating magnetic field.

\begin{tabular}{|c|c|}
\hline Excitation coil-1 & 71 turns, direct current: $2 \mathrm{~A}$ \\
\hline Excitation coil-2 & 71 turns, $500 \mathrm{~Hz}, 0.2 \mathrm{~A}(\mathrm{rms})$ \\
\hline Detection coil & $\begin{array}{l}60 \text { turns, width in } x \text {-direction: } 2.5 \mathrm{~mm} \text {, } \\
\text { thickness in } z \text {-direction: } 2.5 \mathrm{~mm} \text {, } \\
\text { length in } y \text {-direction: } 3.3 \mathrm{~mm}\end{array}$ \\
\hline Lift-off $\left(L_{o}\right)$ & $0.5 \mathrm{~mm}$ \\
\hline Specimen & $\begin{array}{l}\text { SS400 steel plate, } \\
\text { conductivity: } 7.505 \times 10^{6} \mathrm{~S} / \mathrm{m} \text {, } \\
\text { width in } x \text {-direction: } 150 \mathrm{~mm} \text {, } \\
\text { thickness in } z \text {-direction: } 2 \mathrm{~mm} \text {, } \\
\text { length in } y \text {-direction: } 150 \mathrm{~mm}\end{array}$ \\
\hline Opposite-side defect & $\begin{array}{l}\text { width in } x \text {-direction }\left(D_{w}\right): 0.5 \mathrm{~mm} \text {, } \\
\text { depth in } z \text {-direction }\left(D_{d}\right): 1 \mathrm{~mm} \text {, } \\
\text { length in } y \text {-direction }\left(D_{l}\right): 100 \mathrm{~mm}\end{array}$ \\
\hline Nodes and elements & 29008,25920 \\
\hline Convergence criterion & $\begin{array}{l}N-R \text { method: } 1.0 \times 10^{-6} \mathrm{~T} \text {, } \\
\text { ICCG method: } 1.0 \times 10^{-5}\end{array}$ \\
\hline
\end{tabular}

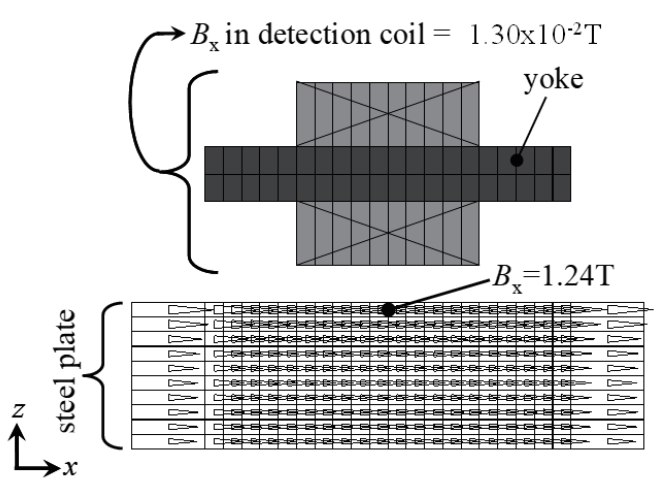

(a)

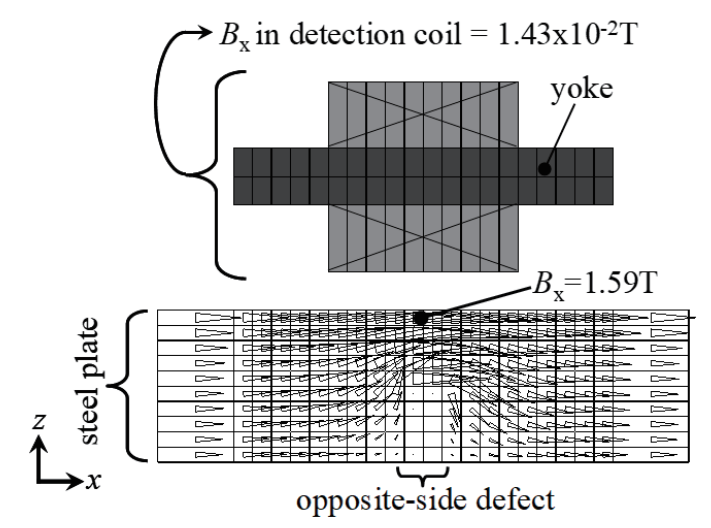

(b)

Fig. 6. Distribution of synthetic magnetic field consisting of static and alternating (500 Hz) magnetic fields inside the steel plate with and without a defect. (a) Without a defect and (b) with a defect.

on the surface steel plate is increased. The peak value of the flux density $B_{x}$ at the $500 \mathrm{~Hz}$ component inside the detection coil is increased from $1.304 \times 10^{-1}$ to $1.427 \times 10^{-2} \mathrm{~T}$ when there is a defect. Moreover, the difference $\Delta B_{x}$ in the flux density in the detection coil with and without the defect is $0.123 \times 10^{-2} \mathrm{~T}$.

As shown in Figs. 5(a) and 6(a), when there are no defects, the low-frequency and highfrequency synthetic flux density $B_{x}$ on the surface layer of the steel plate is lower than the synthetic flux density of the large static and high-frequency magnetic field. This is considered to be because the eddy currents from both the low-frequency and high-frequency magnetic fields cancel the synthetic magnetic field. On the other hand, the difference in the flux density with 
and without defects in the steel plate is larger for the synthetic magnetic field of low and high frequencies than for the synthetic magnetic field of the static and alternating magnetic fields. Similarly, the difference in the leakage magnetic flux with and without defects in the steel plate is larger in the synthetic magnetic field of low and high frequencies than in the synthetic magnetic field consisting of the static and alternating magnetic fields.

Figure 7 shows the absolute value of the flux density inside the steel plate below the detection coil when there is no defect. The horizontal axis in this figure represents the depth from the surface of the steel plate when the synthetic magnetic field of the large static and minute alternating magnetic fields and the synthetic magnetic field of the large low frequency and the minute high frequency are impressed to the steel plate. This figure also shows the distribution of the flux density when only a low-frequency alternating magnetic field of $5 \mathrm{~Hz}$ is applied to the steel plate. The distribution of the flux density is gradually decreased from the surface layer to the opposite side of the steel plate when the alternating magnetic field of $5 \mathrm{~Hz}$ only is impressed to the steel plate. However, the flux density in the steel plate by the excitation frequency of only $5 \mathrm{~Hz}$ is higher than the flux density of the static magnetic field due to the skin effect. Generally, when the alternating magnetic field is applied to the steel plate from the magnetic yoke shaped as shown in Fig. 1(a), the flux density in the surface layer of the steel plate is increased, ${ }^{(13,14)}$ since the flux density is concentrated and distributed on the surface layer of the steel plate by the skin effect. When the excitation frequency is $5 \mathrm{~Hz}$, the flux density inside the steel plate with the thickness of $2 \mathrm{~mm}$ is higher than that of the static magnetic field since the skin depth is about $2.12 \mathrm{~mm}$. Therefore, if there is an opposite-side defect in the steel plate, the leakage flux on the surface of the steel plate will be greater than that of the synthetic magnetic field consisting of the large static magnetic field and the minute alternating magnetic field. On the other hand, when the synthetic magnetic field of the large low frequency and the minute high frequency is applied to the steel plate, the flux density on the surface layer in the steel plate is slightly reduced due to the effects of eddy currents at the two excitation frequencies. This is because the synthetic flux density is canceled by the eddy currents from both the low-frequency and high-frequency flux densities.
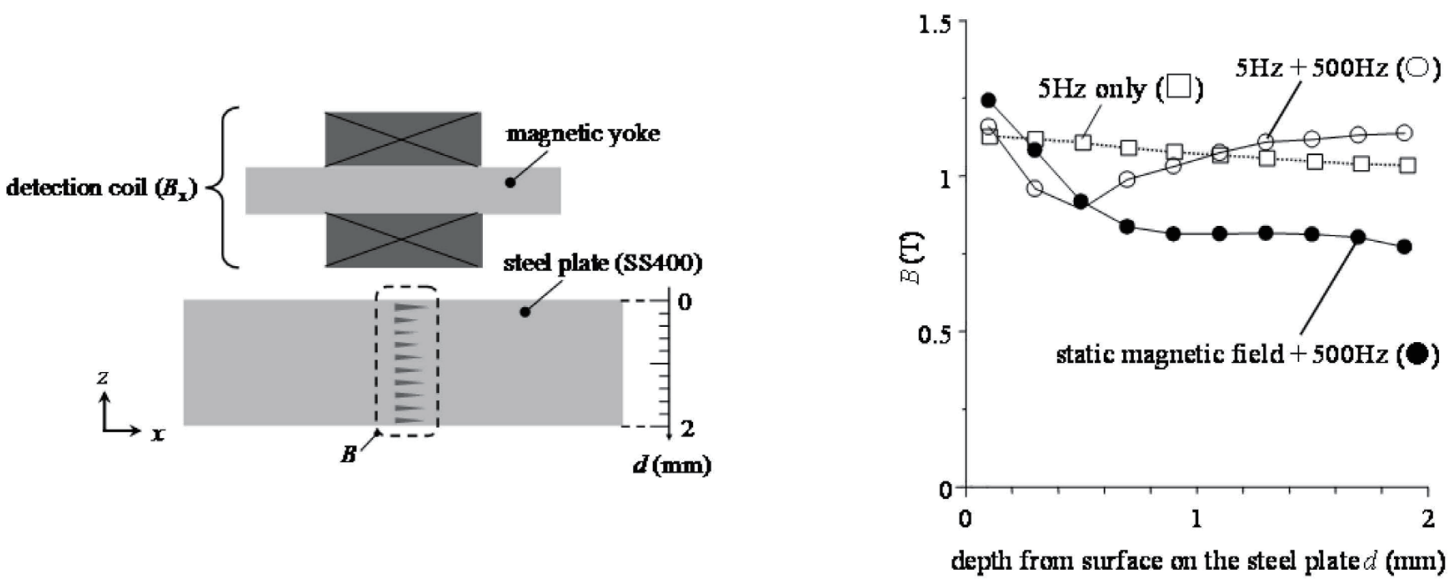

Fig. 7. Distribution of flux density inside the steel plate below the detection coil. 


\section{Verification Experiment and Discussion}

A verification experiment is carried out as shown in Fig. 8. In this experiment, the center of the opposite-side defect in the $x$-direction is set as $x=0 \mathrm{~mm}$, and the inspection sensor is moved in the $x$-direction from -10 to $10 \mathrm{~mm}$ by $0.5 \mathrm{~mm}$ steps, keeping the lift-off at $0.5 \mathrm{~mm}$. The peak value of the flux density $B_{x}$ at the $500 \mathrm{~Hz}$ component inside the detection coil is measured. The excitation conditions are the same as in the 3D nonlinear FEM analysis shown in Table 1. In addition, an opposite-side defect in the steel plate is also measured using the synthetic magnetic field consisting of the large static magnetic field and the minute alternating magnetic field. The direct excitation current for the static magnetic field of excitation coil-1 is set to 142 ampereturns, and the alternating excitation current of the excitation coil-2 is set to 14.2 ampere-turns with a frequency of $500 \mathrm{~Hz}$.

Figure 9 shows the change $\Delta B_{x}$ of the flux density in the detection coil at each measured position. This figure shows that the inspection sensor can detect the opposite-side defect since $\Delta B_{x}$ is increased at the position of the defect. Moreover, when the synthetic magnetic field with the large low frequency and minute high frequency is used, $\Delta B_{x}$ is larger than that when the synthetic magnetic field consisting of the large static magnetic field and the minute alternating magnetic field is used. Figure 10 shows a comparison of the measured and calculated results of

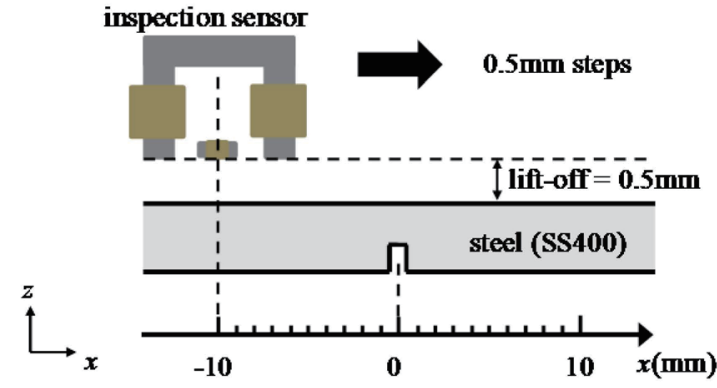

Fig. 8. (Color online) Verification experiment model using proposed.

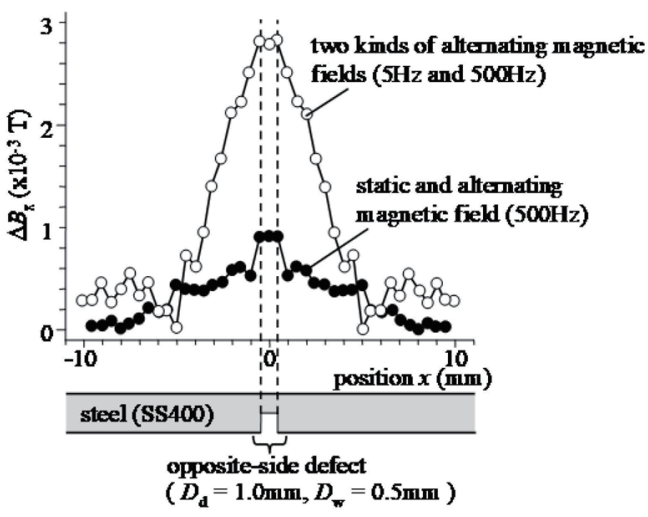

Fig. 9. Inspection results of $\Delta B_{x}$ in the detection coil. inspection coil.

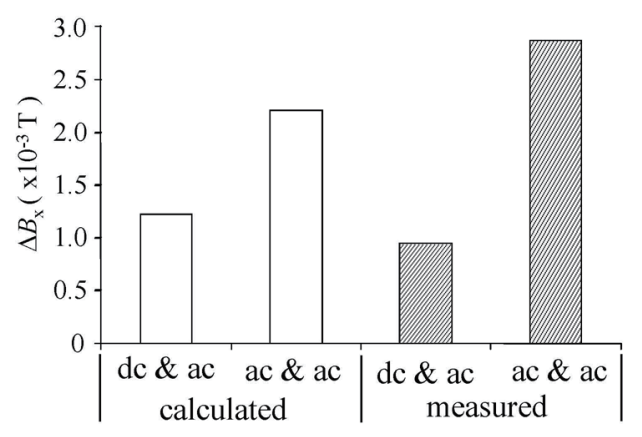

Fig. 10. Calculated and measured inspection results of peak value of $\Delta B_{x}$. 
the peak value of $\Delta B_{x}$ at the position of $x=0 \mathrm{~mm}$. This figure shows that the peak value of $\Delta B_{x}$ of the synthetic magnetic field with the large low frequency and the minute high frequency is higher than that of the synthetic magnetic field consisting of the large static magnetic field and the minute high-frequency alternating magnetic field. Moreover, the measured results show the same tendency as the calculated results.

\section{Conclusions}

The results obtained by this research are summarized as follows:

(1) An opposite-side defect in a thin steel plate is detected by a synthetic magnetic field consisting of a large magnetic field with a low frequency and a minute magnetic field with a high frequency. This is because the synthetic flux density is concentrated in the layer between the opposite-side defect and the surface steel plate when there is a defect, which increases the leakage flux.

(2) The detection signal of the synthetic magnetic field consisting of the large magnetic field with a low frequency and the minute magnetic field with a high frequency is larger than that of a synthetic magnetic field consisting of large static and minute alternating magnetic fields with a high frequency. Since the skin effect of the alternating magnetic field with the low frequency in the synthetic magnetic field using the two different excitation frequencies is manifested within the thickness of the steel plate, the flux density in the entire steel plate is increased. Therefore, if there is an opposite-side defect in the steel plate, the leakage flux on the surface of the steel plate is increased.

The effects of the detection signal on the optimum frequencies used for the synthetic magnetic field and the shape of the defect will be the subject of future research.

\section{References}

1 G. Vertesy, I. Meszaros, and I. Tomas: J. Magn. Magn. Mater. 285 (2005) 335. https://doi.org/10.1016/j. jmmm.2004.08.006

2 Y. Gotoh and N. Takahashi: IEEE Trans. Magn. 44 (2008) 1622. https://jglobal.jst.go.jp/detail?JGLOBAL ID $=200902249602838935$

3 A. Fujii, T. Adachi, and Y. Gotoh: Int. J. Appl. Electromagn. Mech. 52 (2016) 1045. https://doi.org/10.3233/JAE162185

4 Y. Gotoh, K. Sakurai, and N. Takahashi: IEEE Trans. Magn. 45 (2008) 4467. https://doi.org/10.1109/ TMAG.2009.2024894

5 S. Yoshioka, T. Tujigou, and Y. Gotoh: IEEE Trans. Magn. 53 (2017). https://doi.org/10.1109/ TMAG.2017.2656946

6 Y. L. Gui, Y. Tian, and S. Ward: NDT \& E Int. 39 (2006) 367. https://doi.org/10.1016/j.ndteint.2005.10.006

7 Y. Shi, C. Zhang, R. Li, M. Cai, and G. Jia: Sensors 15 (2015) 31036. https://doi.org/10.3390/s151229845

8 A. K. Rostamabad, J. P. Reilly, N. K. Nikolova, J. R. Hare, and S. Pasha: IEEE Trans. Magn. 45 (2009) 3073. https://doi.org/10.1109/TMAG.2009.2020160

9 Y. Gotoh, and N. Takahashi: IEEE Trans. Magn. 42 (2006) 1415. https://ieeexplore.ieee.org/document/1608481

10 Y. Gotoh, and N. Takahashi: IEEE Trans. Magn. 38 (2002) 1209. https://ci.nii.ac.jp/naid/120002305673

11 K. Miyata, K. Ohashi, A. Muraoka, and N. Takahashi: IEEE Trans. Magn. 42 (2006) 1452. https://doi. org/10.1109/TMAG.2006.871430

12 Y. Gotoh and H. Tanaka: IEEE Trans. Magn. 50 (2014) 1622. https://doi.org/10.1109/TMAG.2007.916486

13 Y. Gotoh and N. Takahashi : IEEE Trans. Magn. 40 (2004) 655. https://ieeexplore.ieee.org/document/1284499

14 Y. Gotoh and N. Takahashi: IEEE Trans. Magn. 42 (2006) 1451. https://ieeexplore.ieee.org/document/1608490 